\title{
CORRIGENDUM
}

\section{One-third of Danish hypertrophic cardiomyopathy patients with MYH7 mutations have mutations in rod region}

Lotte Hougs, Ole Havndrup, Henning Bundgaard, Lars Køber, Jens Vuust, Lars Allan Larsen, Michael Christiansen and Paal Skytt Andersen

European Journal of Human Genetics (2005) 13, 694. doi:10.1038/sj.ejhg.5201385

Correction to: European Journal of Human Genetics (2005)

13, 161-165. doi: 10.1038/sj.ejhg.5201310
The title was published incorrectly; the correct title is above. 